\title{
The Bose-Hubbard Model: From Josephson Junction Arrays to Optical Lattices
}

\author{
C. Bruder ${ }^{* 1}$, Rosario Fazio* ${ }^{* * 2}$, and Gerd Schön ${ }^{* * * 3}$ \\ ${ }^{1}$ Department of Physics and Astronomy, University of Basel, Klingelbergstr. 82, 4056 Basel, \\ Switzerland \\ ${ }^{2}$ NEST-INFM \& Scuola Normale Superiore, Piazza dei Cavalieri 7, 56126 Pisa, Italy \\ ${ }^{3}$ Institut für Theoretische Festkörperphysik, Universität Karlsruhe, 76128 Karlsruhe, Germany
}

Received 15 June 2005

Key words Bose-Hubbard model, Josephson junction arrays, optical lattices PACS 74.81.Fa,05.30.Jp,73.43.Nq

Dedicated to Bernhard Mühlschlegel on the occasion of his 80th birthday

The Bose-Hubbard model is a paradigm for the study of strongly correlated bosonic systems. We review some of its properties with emphasis on the implications on quantum phase transitions of Josephson junction arrays and quantum dynamics of topological excitations as well as the properties of ultra-cold atoms in optical lattices.

\section{Introduction}

For several decades major activities of the physics community have been devoted to the study of the properties of strongly interacting physical systems. Despite substantial progress, both in experiment and theory, still many important issues lack a proper understanding. One of the routes to progress in this direction is the creation of artificial structures with tunable parameters that can reproduce the properties of strongly interacting quantum systems. In this note we briefly review the successful story of some examples of "custom-made" strongly interacting boson systems.

Experiments performed in the early 1980s on granular superconductors had suggested a metalinsulator transition at low temperatures which depends on the value of the normal state conductance of the network $[1,2,3]$. B. Mühlschlegel, who had been investigating properties of Josephson junction arrays including disorder [4] noticed the potential importance of these findings and stimulated further work in this direction [3]. Later it became possible, in particular in the groups of J.E. Mooij in Delft and M. Tinkham in Harvard, to fabricate regular Josephson Junction Arrays (JJAs) with island and junction dimensions in the submicron regime. Transport studies revealed a wealth of phenomena, which in turn stimulated further theoretical activities. Some of the most notable findings were the discovery of a quantum phase transition, persisting to zero temperature, between a superconducting and an insulating phase. It arises as a consequence of a duality between charges and vortices, which also explains the universality of the conductance at the transition point $[5,6,7]$. Similarly exciting was the observation that the topological excitations, in this case the vortices, are quantum degrees of freedom with corresponding dynamics [8].

* email: christoph.bruder@unibas.ch

** email: fazio@sns.it

*** Corresponding author: email: schoen@tfp.physik.uni-karlsruhe.de 
The studies of Josephson junction arrays have found, in the middle of the 1990s, an extension in a new and unexpected direction: the area of ultra-cold quantum gases [9]. It was proposed theoretically [10] and verified experimentally [11] that bosons loaded in an optical lattice could undergo a superfluid-insulator phase transition at zero temperature. On one hand, as we will review briefly below, the superconductor-insulator transition observed in JJAs and the superfluidinsulator transition in optical lattices have the same origin. They are caused by the competition between the trend to global coherence, due to the hopping of bosonic particles, and the tendency towards localization induced by the strong interactions. On the other hand, despite the strong analogies, the two model systems allow the investigation of different physical observables. The charged bosons in Josephson arrays are studied by means of transport measurements. In the optical lattices we have neutral bosons. Standard tools to study them are expansion experiments (the trap is switched off, and the expanding cloud is investigated by taking optical absorption pictures) and light scattering.

The present paper is intended to provide a brief review and comparison of the two types of systems. It is organized as follows: we will first introduce the Bose-Hubbard model, a paradigm for strongly interacting bosons, and discuss its phase diagram. We will then give a brief introduction to Josephson junction arrays and optical lattices, show how to describe those systems by means of the Bose-Hubbard model, and discuss a few of the specific properties

\section{The Bose-Hubbard model}

Both Josephson junction arrays and optical lattices are described in certain limits by the BoseHubbard model,

$$
H=\frac{1}{2} \sum_{i j} n_{i} U_{i j} n_{j}-\mu \sum_{i} n_{i}-t \sum_{\langle i j\rangle} b_{i}^{\dagger} b_{j}+\text { h.c. }
$$

Here, $b_{i}^{\dagger}, b_{i}$ are creation and annihilation operators for bosons on the $i$-th site and $n_{i}=b_{i}^{\dagger} b_{i}$ their number operators. The coupling $U_{i j}$ describes the interaction between bosons $\left(U_{i i} \equiv U\right), \mu$ is the chemical potential, and $t$ the hopping matrix element. The hopping, described by the last term in Eq. (1), is restricted to nearest neighbors, and each (directed) bond is counted once. The range and nature of the boson interaction depends on the system considered. It is typically on-site in the case of optical lattices while it may be long-range in the case of JJAs.

A qualitative understanding of the zero-temperature phase diagram can be obtained by considering the limiting cases in which one of the two coupling energies ( $t$ or $U$ ) dominates. If the hopping dominates the lowest energy state is a condensate of bosons delocalized over the lattice. In contrast, if the interaction dominates, in the ground state each site is occupied by a well-defined number of bosons. In order to put an extra boson on a given site, one has to overcome an energy of the order of $U$. The strong local repulsion prevents the establishing of global coherence, and below a critical value of the ratio $t / U$, even at zero temperature, the system is a Mott insulator.

For zero hopping the Mott gap (the energy to add an extra boson on a uniform background of $n$ bosons per site) is given by

$$
E_{G}=n \sum_{j} U_{i j}+\frac{1}{2} U_{i i}-\mu
$$

Evidently at certain values of the chemical potential the gap shrinks to zero, and the energy to add (or remove) a particle to (from) a given site vanishes. This implies that a purely local repulsion 
does not suppress the condensation, and the superfluid phase persists to arbitrary low values of the hopping parameter. A sketch of the resulting phase diagram is shown in Fig. 1. It consists of a series of Mott-insulating lobes [12]. The nature of the superfluid-insulator transition differs, depending where the phase boundary is crossed. At the tip of the lobe the universality class is that of a $d+1$-dimensional classical $X Y$ model, and the dynamical critical exponent is $z=1$. Away from the tip, where particle-hole symmetry is broken, the critical exponent is $z=2$.

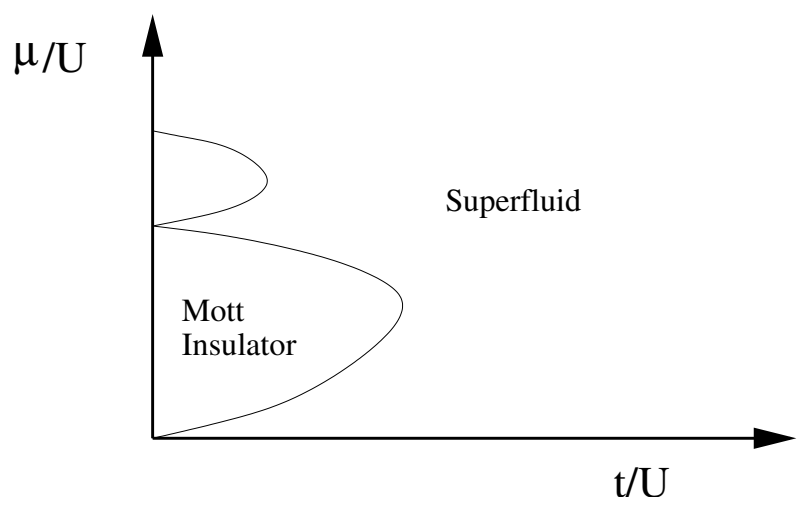

Fig. 1 Zero-temperature phase diagram of the Bose-Hubbard model. Inside the lobes the system is in a Mott insulator state. The different lobes (only the first two are drawn in the figure) have different fillings.

The Bose-Hubbard model was successfully applied to model JJAs, granular superconductors, ultra-thin amorphous films and, more recently, optical lattices. In the following, we will discuss only arrays and optical lattices where disorder is absent or can be introduced in a controlled way.

\section{Josephson Junction Arrays}

A Josephson junction array consists of a regular network of superconducting islands weakly coupled by tunnel junctions. The first artificially fabricated JJAs were realized twenty years ago [13] as part of an effort to develop an electronics based on superconducting devices. The investigation of JJAs produced a wealth of classical phenomena $[1,14,15]$. The arrays proved to be an ideal model system in which classical phase transitions, frustration effects, classical vortex dynamics, as well as non-linear dynamics and chaos could be studied in a controlled way.

Josephson junction arrays in the quantum regime were first fabricated in Delft [16]. It became possible to enter this regime due to the progress in lithography that allowed realizing submicron junctions with a high tunneling resistance (of the order of a few $\mathrm{k} \Omega$ ). The parameters of the array (like the shape of the islands, the thickness of the oxide barrier, etc.) can be made highly uniform across the whole array. The largest samples realized consisted of about $10^{4}$ junctions.

What is the appropriate model to describe a JJA? The coupling strength between adjacent islands is determined by the Josephson energy $E_{J}$. Quantum effects in Josephson arrays come into play whenever the charging energy (associated with non-neutral charge configurations of the islands) is comparable with the Josephson coupling. A characteristic energy scale that characterizes the strength of the electrostatic interaction is given by $E_{C}=e^{2} / 2 C$, where $C$ is geometrydependent effective capacitance. In order to keep dissipative effects at a low level the junction resistance should be larger than the quantum of resistance $R_{Q}=h / 4 e^{2}$. The electrostatic energy is determined by the capacitance matrix $C_{i j}$ and applied gate voltages (if present). Generally one only considers the junction capacitances $C$ and the capacitance to the ground $C_{0}$. In the case of two-dimensional square lattices the inverse capacitance matrix and interaction energy for two 
charges placed in islands $i$ and $j$ (with coordinates $\mathbf{r}_{i}$ and $\mathbf{r}_{j}$ ) is given by

$$
U_{i j}=\frac{e^{2}}{2} \int \frac{d \mathbf{k}}{4 \pi^{2}} \frac{\exp \left(i \mathbf{k} \cdot\left(\mathbf{r}_{i}-\mathbf{r}_{j}\right)\right)}{C_{0}+2 C\left(1-\cos k_{x}\right)+2 C\left(1-\cos k_{y}\right)} .
$$

For $C \gg C_{0}$ the interaction decays slowly (logarithmically) up to distances of the order of the screening length $\lambda \sim \sqrt{C / C_{0}}$ (in units of the lattice constant) and then dies out exponentially.

Adding the Josephson coupling, which describes the Cooper pair tunneling, and the interaction of Cooper pair charges we arrive at the Quantum Phase Model (QPM) Hamiltonian:

$$
H=\frac{1}{2} \sum_{i, j}\left(q_{i}-q_{\mathrm{x}}\right) U_{i j}\left(q_{j}-q_{\mathrm{x}}\right)-E_{J} \sum_{<i, j>} \cos \left(\phi_{i}-\phi_{j}\right) .
$$

Here $2 e q_{i}$ is the net charge on the $i$-th island. An external gate voltage $V_{\mathrm{x}}$ contributes to the energy via the induced charge $q_{\mathrm{x}}=\sum_{j} C_{i j} V_{\mathrm{x}} / 2 e$. Quantum mechanics enters through the commutation relation between charge and phase operators

$$
\left[q_{i}, e^{i \phi_{j}}\right]=\delta_{i j} e^{i \phi_{j}}
$$

The connection between the Bose-Hubbard and the Quantum Phase model can be seen by writing the field $b_{i}$ in terms of its amplitude and phase and subsequently approximating the amplitude by its average (the mapping becomes more accurate as the average number of bosons per site increases). This procedure leads to the following identification of the quantities in the two formulations: $b_{i} \rightarrow \exp \left(-i \phi_{i}\right),\langle n\rangle t \rightarrow E_{J}, n_{i} \rightarrow q_{i}$, and $\mu+U \rightarrow \sum_{j} U_{i j} q_{\mathrm{x}, j}$.

The two contributions in the Hamiltonian Eq. (4) favor different types of ground states. The Josephson energy tends to establish phase coherence. On the other hand, the charging energy favors charge localization on each island and therefore tends to suppress superconducting coherence. This interplay becomes evident if one recalls the Josephson relation

$$
\frac{d \phi_{i}}{d t}=\frac{2 e}{\hbar} V_{i}=\frac{2 e}{\hbar} C_{i j}^{-1} Q_{j}
$$

A constant charge (in time) on the islands implies strong fluctuations in the phases. On the other hand, phase coherence leads to strong fluctuations in the charge.

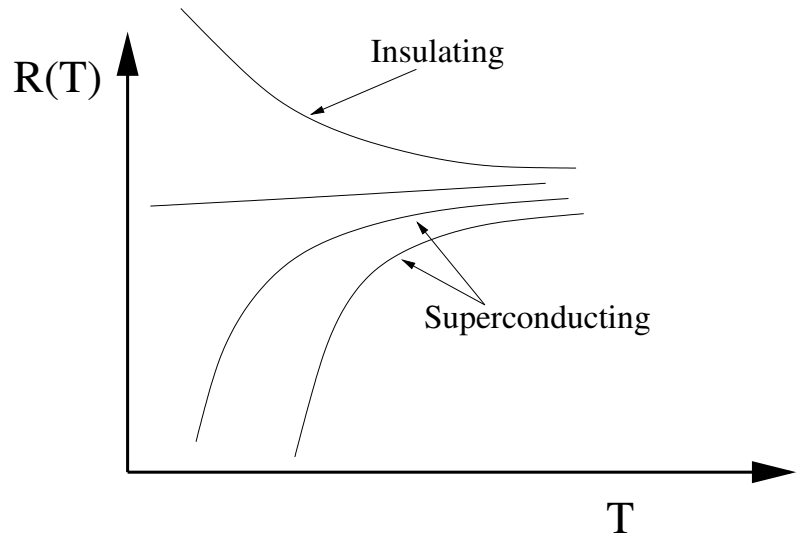

Fig. 2 A schematic plot of the resistance of a Josephson array as a function of temperature for different samples. At the critical value of $E_{J} / E_{C}$ the system becomes insulating. 
The properties of Josephson arrays in the quantum regime have been reviewed in Ref. [7]. Here we recall some characteristics of the superconductor-insulator transition. The transition is marked by a differing behavior of the resistance as a function of temperature for different values of the ratio $E_{J} / E_{C}$. A schematic plot is given in Fig. 2. Samples for which the Josephson coupling dominates undergo at finite temperature a transition to a superconducting state. Below this critical temperature the array is globally coherent. However, on lowering the ratio $E_{J} / E_{C}$ at some critical value the resistance increases when lowering the temperature. This upturn of the resistance signals the existence of an insulating state at zero temperature. The striking fact is that the whole array is insulating despite the fact that each island is still superconducting!

Charge-vortex duality. The properties of a Josephson array are to a large extent characterized by its topological excitations: charges and vortices. A powerful way to highlight their role is to map the QPM, Eq. (4) onto a model which is explicitly expressed in terms of charge and vortex degrees of freedom $[17,18,19,20]$. Indeed there is a dual transformation relating the classical vortex limit, $E_{J} \gg E_{C}$, to the opposite charge limit, $E_{J} \ll E_{C}$. The duality is most perfect in the case $C_{0} \ll C$, which can be realized to some degree of approximation in suitable arrays. The interaction between charges on islands is then logarithmic, analogous to vortex interactions in classical, superconducting arrays. The charges form a 2D Coulomb gas and are expected to undergo a BKT transition at $T_{c h}^{(0)} \sim E_{C}[21]$.

To display the duality the partition function of a JJA can be expressed as a sum over charge $q$ and vortex $v$ configurations $Z=\sum_{[q, v]} e^{-S\{q, v\}}$. The effective action $S\{q, v\}$ reads

$$
\begin{aligned}
& S\{q, v\}=\int_{0}^{\beta} d \tau \quad \sum_{i, j} \quad\left\{\frac{1}{2} q_{i}(\tau) U_{i j} q_{j}(\tau)+\pi E_{J} v_{i}(\tau) G_{i j} v_{j}(\tau)\right. \\
&\left.+\quad i q_{i}(\tau) \Theta_{i j} \dot{v}_{j}(\tau)+\frac{1}{4 \pi E_{J}} \dot{q}_{i}(\tau) G_{i j} \dot{q}_{j}(\tau)\right\} .
\end{aligned}
$$

This action describes two coupled Coulomb gases of two integer-valued fields, $q$ and $v$. For clarity we have used a continuous time representation, but the path integral is defined on a discretized time lattice. The charges interact via the electrostatic interaction (first term) defined in Eq. (3). The interaction among the vortices (second term) is described by the kernel $G_{i j}$, which is the Fourier transform of $k^{-2}$. At large distances $r_{i j} \equiv\left|\mathbf{r}_{i}-\mathbf{r}_{j}\right| \gg 1$ it depends logarithmically on the distance: $G_{i j} \sim-\frac{1}{2} \ln r_{i j}$. The third term describes the coupling between the topological excitations, i.e., between charges and vortices. The function

$$
\Theta_{i j}=\arctan \left(\frac{y_{i}-y_{j}}{x_{i}-x_{j}}\right)
$$

represents the vortex-phase configuration at site $i$ when its center is placed at the site $j$. The coupling has a simple physical interpretation: a change of vorticity at site $j$ produces a voltage at site $i$ which is felt by the charge at this location. The last term $\dot{q} G \dot{q}$ stems from the spin-wave contribution to the charge-correlation function.

The effective action in Eq. (7) shows a high degree of symmetry between the vortex and charge degrees of freedom. In particular, in the limit $C_{0} \ll C$ the inverse capacitance matrix has the same functional form as the kernel describing vortex interactions:

$$
U_{i j}=\frac{2}{\pi} E_{C} G_{i j} .
$$

Hence charges and vortices are dual. There is a critical point for which the system is self-dual with respect to interchanging them:

$$
\frac{E_{J}}{E_{C}}=\frac{2}{\pi^{2}}
$$


The duality is strict for vanishing self-capacitance and in the absence of the spin-wave duality breaking term $(\dot{q} G \dot{q})$ in Eq. (7). This latter term is irrelevant at the critical point, i.e., it merely shifts the transition point. However, it has important implications for the dynamical behavior.

Vortex dynamics. The coupled-Coulomb-gas representation, Eq. (7), displays the coupling between charges and vortices. In the extreme limits, $E_{J}=0$ or $E_{C}=0$, only one or the other needs to be considered. In the limit $E_{J} \gg E_{C}$, we still obtain an effective action for the vortices only, but they are influenced by the charging effects. In the considered limit the charges are fluctuating strongly and can be integrated out from the partition function [22]. In the limit where the junction capacitance dominates $C \gg C_{0}$ the result is

$$
S[v]=\int_{0}^{\beta} d \tau \sum_{i, j}\left[\frac{\pi}{8 E_{C}} \dot{v}_{i}(\tau) G_{i j} \dot{v}_{j}(\tau)+\pi E_{J} v_{i}(\tau) G_{i j} v_{j}(\tau)\right] .
$$

The vorticity at each space-time point takes the values 0 or \pm 1 and changes in discrete steps. Vortices can be created and annihilated in pairs. In some limits it is sufficient to assume that the vortices move in a continuous way. Then the resulting effective action for a single vortex reads

$$
\begin{aligned}
S_{e f f} & =\frac{1}{2} \sum_{a, b=x, y} \int d \tau d \tau^{\prime} \dot{r}^{a}(\tau) M_{a b}\left[\mathbf{r}(\tau)-\mathbf{r}\left(\tau^{\prime}\right), \tau-\tau^{\prime}\right] \dot{r}^{b}\left(\tau^{\prime}\right), \\
M_{a b} & =\sum_{j k} \nabla_{a} \Theta\left(\mathbf{r}(\tau)-\mathbf{r}_{j}\right)\left\langle q_{j}(\tau) q_{k}\left(\tau^{\prime}\right)\right\rangle \nabla_{b} \Theta\left(\mathbf{r}_{k}-\mathbf{r}\left(\tau^{\prime}\right)\right),
\end{aligned}
$$

where the function $\Theta(\mathbf{r})$ has been defined in Eq. (8). Thus, the vortex dynamics is governed by the charge-charge correlation, which depends on the full coupled charge-vortex gas. The effective action Eq. (12) describes dynamical vortex properties in the whole superconducting region and is therefore a good starting point for the investigation of vortex properties down to the S-I transition. The expression given in Eq. (12) reproduce the Eckern-Schmid mass [8] in the classical limit where $E_{J} \gg E_{C}$. In this region of the phase diagram the charges may be considered to be continuous variables and vortex fluctuations may be neglected so that the charge-charge correlation reads

$$
\langle q q\rangle_{k, \omega_{\mu}}=E_{J} k^{2} \frac{1}{\left(\omega_{\mu}^{2}+\omega_{k}^{2}\right)} \quad \text { with } \quad \omega_{k}^{2}=\frac{4 e^{2} E_{J}}{C_{0}} \frac{k^{2}}{1+\lambda^{2} k^{2}} .
$$

The spin-wave dispersion is described by $\omega_{k}$. For long-range Coulomb interactions it is optical, $\omega_{k}=\omega_{p}$, whereas for on-site interactions it is $\omega_{k}=\bar{\omega}_{p} k$. Here $\bar{\omega}_{p}=\sqrt{4 e^{2} E_{J} / C_{0}}$ is the plasma frequency for the case of on-site Coulomb interactions.

In the limit of low velocities $\dot{r}(\tau)$ the action (12) reduces to that of a free particle. The corresponding adiabatic vortex mass $M_{v}=\int_{0}^{\beta} d \tau M_{x x}(0, \tau)$ becomes in the classical limit

$$
M_{v}=\frac{\pi^{2}}{4 E_{C}}+\frac{\pi C_{0}}{4 e^{2}} \ln (L) .
$$

Thus both $C_{0}$ and $C$ yield a contribution to the mass. The self-capacitance contribution depends on the system size $L$. For generic sample sizes and capacitance ratio's the size-dependent contribution is smaller than the Eckern-Schmid mass [8].

A moving vortex experiences experiences a spin-wave damping that may also be calculated from Eq. (12). Varying the vortex coordinate $r^{a}(\tau)$ in Eq. (12) yields the equation of motion

$$
2 \pi \epsilon_{a b} I_{b} / I_{c}=\partial_{\tau} \int d \tau^{\prime} M_{a b}\left(r(\tau)-r\left(\tau^{\prime}\right), \tau-\tau^{\prime}\right) \dot{r}^{b}\left(\tau^{\prime}\right)
$$


$\left(\epsilon_{x x}=\epsilon_{y y}=0, \epsilon_{x y}=-\epsilon_{y x}=1\right)$. Its constant-velocity solutions in the presence of an external current determine the non-linear relation between driving current and vortex velocity and, hence, the current-voltage characteristics [23]. The relevant information is contained in the real part of Eq. (15), which for constant vortex velocity reads

$$
I^{y} / I_{c r}=\frac{v}{4} \int d \omega \int d^{2} k \frac{k_{y}^{2}}{k^{2}} \delta\left(\omega-v k_{x}\right)\left[\delta\left(\omega-\omega_{k}\right)+\delta\left(\omega-\omega_{k}\right)\right] .
$$

The delta-functions express the spin-wave dispersion and the vortex dispersion, respectively. The overlap integral determines the amount of dissipation a moving vortex suffers from coupling to spin-waves.

Besides the experimental verification of the mass term in the equation of motion [24, 25] considerable interest focused on the direct observation of the ballistic motion. Ballistic vortex motion has not only been observed in long continuous junctions [26], where energy barriers for cell-to-cell motion and spin-wave coupling are absent, but also in discrete $1 \mathrm{D}$ arrays $[27,28,29]$ and in 2D aluminum arrays [30].

Magnus force. In addition to the Lorentz force, due to the external current (1.h.s. of Eq. (15)), a vortex is subject to a Magnus force which acts transverse to vortex velocity. The study of the Magnus force in superfluids has a long and controversial history. A detailed discussion can be found in Ref. [31] and references therein. In Josephson arrays, in presence of a gate to the ground plane, particle-hole symmetry is broken. A vortex feels a Magnus force $[18,32]$ given by $\left(\Phi_{0}=h c / 2 e\right)$

$$
\mathbf{F}=2 e q_{x} \Phi_{0} \hat{\mathbf{z}} \times \dot{\mathbf{r}}
$$

Note that the force is proportional to the physical charge and not to the superfluid density. As a result of the combined effect of the Magnus force and the Lorentz force, the vortices move at a certain angle, the Hall angle, with respect to the current. Its measurement yields information on the various sources of dissipation. Assuming for simplicity that the vortex moving with constant velocity $\mathbf{v}=\left[v_{x}, v_{y}\right]=\left[v \cos \theta_{H}, v \sin \theta_{H}\right]$ experiences a dissipation characterized by the strength $\eta$ we arrive in the stationary limit at the relations

$$
\begin{aligned}
\eta v \cos \theta_{H} & =I_{y} \Phi_{0}-Q_{x} v \Phi_{0} \sin \theta_{H} \\
\eta v \sin \theta_{H} & =Q_{x} v \Phi_{0} \cos \theta_{H} .
\end{aligned}
$$

It lead to the resistance tensor

$$
R_{x x}=R_{x x}=\frac{\Phi_{0}^{2} / \eta}{1+\left(Q_{x} \Phi_{0} / \eta\right)^{2}} \quad, \quad R_{x y}=-R_{y x}=\frac{Q_{x} \Phi_{0}^{3} / \eta^{2}}{1+\left(Q_{x} \Phi_{0} / \eta\right)^{2}}
$$

and to the Hall angle $\theta_{H}$

$$
\tan \theta_{H}=\frac{Q_{x} \Phi_{0}}{\eta}
$$

In Ref. [18] the Magnus force was obtained from the QPM, implying that only the external charge enters in determining the Hall angle. A reexamination of the problem by Makhlin and Volovik [33] related the (apparent) absence of the Hall angle to the near exact cancellation of the Magnus force with the spectral-flow force. By deriving the effective action from the BCS Hamiltonian, Volovik [34] showed that the offset charges, contributing to the Hall angle, have two different physical origins. In addition to the one stemming from the coupling to the ground plane, there is an additional contribution which depends on the particle-hole asymmetry of the spectrum. 
This latter term is of the order of the small factor $\left(\Delta / E_{F}\right)^{2}$, with $E_{F}$ being the Fermi energy. This confirms the expectation that the Hall angle should be small in Josephson arrays [35].

In experiments on classical Josephson arrays the Hall angle is usually found to be very small (see e.g. Ref. [36]). In quantum Josephson arrays Hall measurements have been performed by the Chalmers group [37].

\section{Optical Lattices}

Following the work of Jaksch et al. [10], ultra-cold bosonic atoms in optical lattices have been suggested as another important realization of the Bose-Hubbard model. The experimental test of the Mott insulator - superfluid transition by Greiner et al. [11] has paved the way to study strongly correlated phenomena in trapped cold atomic gases, see [38] for a review. An optical lattice can be realized by using counter-propagating laser fields. At large blue detuning, they provide a conservative potential of the form (for simplicity, we consider the two-dimensional case)

$$
V_{p}(\mathbf{r})=V_{0}\left(\sin ^{2} a x+\sin ^{2} a y\right),
$$

where $V_{0}$, the strength of periodic potential is controlled by the intensity of the lasers whereas the lattice constant $a$ by means of their wavelength.

In the following we briefly recall the leading steps, following the original derivation in the paper by Jaksch et al., that lead to the mapping onto the Bose-Hubbard model. A system of interacting bosons which is subject to a periodic potential $V_{\mathrm{p}}(\mathbf{r})$ and to a trapping potential $V_{\text {ext }}(\mathbf{r})$ and interacting via a potential $V_{\text {int }}\left(\left|\mathbf{r}-\mathbf{r}^{\prime}\right|\right)$ is described by the Hamiltonian

$$
\begin{aligned}
\mathcal{H} & =\int d^{2} \mathbf{r} \hat{\Psi}^{\dagger}(\mathbf{r})\left[\frac{\mathbf{p}^{2}}{2 M}+V_{\mathrm{p}}(\mathbf{r})+V_{\mathrm{ext}}(\mathbf{r})\right] \hat{\Psi}(\mathbf{r}) \\
& +\frac{1}{2} \int d^{2} \mathbf{r} \int d^{2} \mathbf{r}^{\prime} \hat{\Psi}^{\dagger}(\mathbf{r}) \hat{\Psi}^{\dagger}\left(\mathbf{r}^{\prime}\right) V_{\mathrm{int}}\left(\left|\mathbf{r}-\mathbf{r}^{\prime}\right|\right) \hat{\Psi}\left(\mathbf{r}^{\prime}\right) \hat{\Psi}(\mathbf{r}) .
\end{aligned}
$$

Here $\hat{\Psi}(\mathbf{r})$ and $\hat{\Psi}^{\dagger}(\mathbf{r})$ are field operators satisfying the commutation relations $\left[\hat{\Psi}(\mathbf{r}), \hat{\Psi}^{\dagger}\left(\mathbf{r}^{\prime}\right)\right]=$ $\delta^{(3)}\left(\mathbf{r}-\mathbf{r}^{\prime}\right)$.

It is convenient to decompose the field operator $\hat{\Psi}(\mathbf{r})$ in the following Wannier basis,

$$
\hat{\Psi}(\mathbf{r})=\sum_{n, \mathbf{r}_{i}} w_{n}\left(\mathbf{r}-\mathbf{r}_{i}\right) \hat{b}_{n}\left(\mathbf{r}_{i}\right),
$$

where $\hat{b}_{n}\left(\mathbf{r}_{i}\right)\left(\hat{b}_{n}^{\dagger}\left(\mathbf{r}_{i}\right)\right)$ destroys (creates) a boson at the lattice site $\mathbf{r}_{i}$ in the $n$-th band described by the Wannier function $w_{n}(\mathbf{r})$.

If the external fields are sufficiently weak and in the absence of band crossings, the band index $n$ can be taken to be a constant of the motion. Under these assumptions Eq. (23) can be restricted to the lowest band. This maps the continuous model (22) to the Bose-Hubbard Hamiltonian, with the coupling parameters given by

$$
t=-\int d^{2} \mathbf{r} w^{*}\left(\mathbf{r}-\mathbf{r}_{i}\right)\left[-\hbar^{2} \nabla_{\mathbf{r}}^{2} / 2 M+V_{\mathrm{p}}(\mathbf{r})\right] w\left(\mathbf{r}-\mathbf{r}_{j}\right),
$$

where $i$ and $j$ are nearest neighbors,

$$
U_{i j}=\int d^{2} \mathbf{r} \int d^{2} \mathbf{r}^{\prime}\left|w\left(\mathbf{r}-\mathbf{r}_{i}\right)\right|^{2} V_{\mathrm{int}}\left(\left|\mathbf{r}-\mathbf{r}^{\prime}\right|\right)\left|w\left(\mathbf{r}^{\prime}-\mathbf{r}_{j}\right)\right|^{2},
$$

and

$$
\mu=\int d^{2} \mathbf{r} V_{\mathrm{ext}}(\mathbf{r})\left|w\left(\mathbf{r}-\mathbf{r}_{i}\right)\right|^{2} .
$$


In the presence of an inhomogeneous external potential $V_{\text {ext }}$, we assume that it varies slowly enough such that it makes sense to define a local chemical potential and to apply the BoseHubbard model locally. Usually, the system will then be characterized by coexisting superfluid and Mott-insulating regions.

The long-range phase coherence of cold bosons in an optical lattice can be directly tested by observing a multiple matter-wave interference pattern after ballistic expansion when all external trapping potentials are switched off in a time-of-flight measurement. Phase-coherent matter waves originating from different lattice sites overlap and interfere with each other. Narrow peaks in the momentum distribution, that are due to the periodicity of the lattice, and a constant macroscopic phase difference across the lattice sites become visible [39, 40, 41].
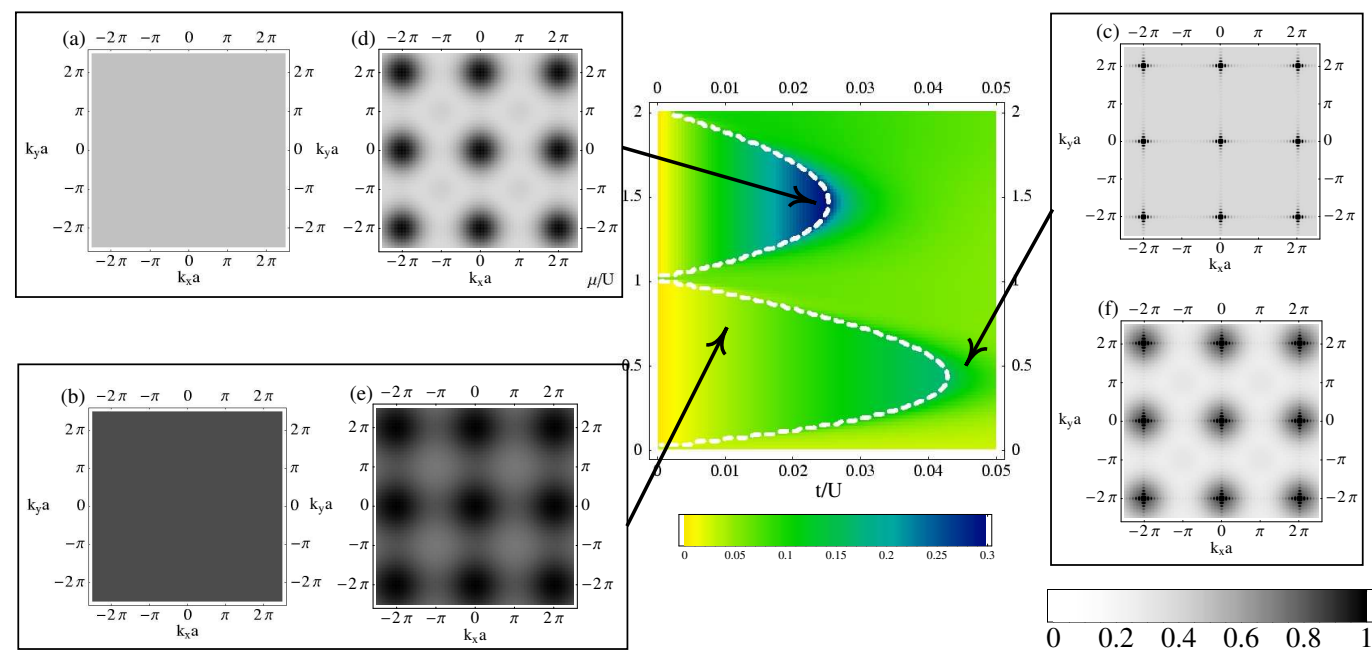

Fig. 3 The central figure shows the correction to the one-particle density matrix $\rho_{i, i+1}$ in second-order perturbation theory as a function of $\mu / U$ and $t / U$. Plots (a) - (f) display the resulting momentum distribution without the Wannier form factor, $\rho(\mathbf{k}) /|w(\mathbf{k})|^{2}$, calculated for a $2 \mathrm{D}$ lattice with $25 \times 25$ lattice sites. (a)-(c) are the Gutzwiller mean-field results, and (d)-(f) are calculated using second-order perturbation theory. Arrows indicate the position of the respective plots in the $(\mu / U, t / U)$ phase diagram. The parameters used are: $\mu / U=1.5, t / U=0.0225$ for (a) and (d); $\mu / U=0.75, t / U=0.01$ for (b) and (e); and $\mu / U=0.5, t / U=0.044$ for (c) and (f). The gray-scales of plots belonging to the same parameter set are identical. Expansion patterns (a),(b),(d), and (e) are normalized to the peak maximum; (c) and (f) are normalized to $1 / 20$ of the peak maximum.

An approximate way to study the transition and to calculate the momentum distribution function was described in [42] (for earlier work in this direction, see [43]) employing a Gutzwiller ansatz plus a perturbation expansion in the hopping in order to take into account the correlations neglected in the (mean-field) Gutzwiller trial wave function. The mean-field Gutzwiller ansatz neglects correlations between different lattice sites. It predicts a one-particle density matrix for the Mott phase that is proportional to a delta function and to a delta function plus a constant in the superfluid phase. Correspondingly, the momentum distribution function in the Mott phase is constant, i.e., the Gutzwiller approach fails to predict the interference patterns discussed before. Systematic corrections to the Gutzwiller state can then be calculated by perturbation theory in the hopping parameter $t$. 
The single-particle density matrix $\rho_{j i}=\left\langle\hat{b}_{n}^{\dagger}\left(\mathbf{r}_{i}\right) \hat{b}_{n}\left(\mathbf{r}_{j}\right)\right\rangle$ is of particular importance as it describes the correlation between the different lattice sites. The correlation function $\rho_{i j}$ shows off-diagonal long-range order in the superfluid state (in dimensions $d>1$ ), in contrast to the Mott phase where $\rho_{i j}$ decays exponentially.

The experimental observation [11] of the Mott transition relies on the different behavior of the density matrix in the Mott and superfluid regimes, which can be visualized by taking absorption pictures of the freely expanding atomic cloud. Assuming that the expansion time is long enough and that the gas is dilute enough (such that atom-atom interactions can be neglected during the expansion), the shape of the cloud reflects the initial momentum distribution $\rho(\mathbf{k})$, which is directly given by the Fourier-transform of the density matrix $\rho_{i j}$ :

$$
\rho(\mathbf{k})=|w(\mathbf{k})|^{2} \sum_{i, j=1}^{M} \rho_{i j} e^{i \mathbf{k}\left(\mathbf{r}_{i}-\mathbf{r}_{j}\right)} .
$$

Here, $w(\mathbf{k})$ is the Fourier transform of the Wannier functions $w(\mathbf{r})$ describing the wavefunction of a single lattice site. The presence of the factor $w(\mathbf{k})$ in Eq. (27) provides a cutoff at high momenta.

The mean-field results for $\rho_{i j}$ describe the different long-range behavior in the Mott and superfluid phase. For a homogeneous lattice the correlation function calculated from the Gutzwiller wavefunction gives $\rho_{i i}=n_{i}$ for the diagonal elements and then drops instantly to $\rho_{i j}=$ const. for $i \neq j$. Short-range correlations, however, are not reproduced by the Gutzwiller approach. This deviation is particularly severe in the Mott phase, where the mean-field result predicts a flat momentum distribution, whereas the short-range correlations (i.e. the exponential decay of $\rho_{i j}$ ) yield smooth bumps in the expansion pattern. These can be distinguished from the $\delta$-peaks of the superfluid only after a sufficiently long expansion time.

Applying perturbation theory improves the structure-less Gutzwiller correlation function. It reproduces the correct slope for the off-diagonal decay and lacks only the wrong offset from the mean field. Thus, the perturbative approach represents a qualitative improvement on the Gutzwiller result.

The results of this approach for the momentum distributions are shown in Fig. 3 in the different regions of the phase diagram $[42,44]$. As an example we discuss a set of momentum distributions $\rho(\mathbf{k}) /|w(\mathbf{k})|^{2}$ for a homogeneous 2D lattice, Fig. 3a-f, and compare the Gutzwiller results to those improved by perturbation theory (PT). The improved PT versions, Figs. 3d-f, show much finer structures than the mean-field results, Fig. 3a-c. PT predicts broad peaks in the MI regions down to very small values of $t / U$, Fig. 3e, whereas the Gutzwiller result without PT shows a structure-less flat distribution for the whole MI region, Fig. 3a,b. Naturally, the modifications of $\rho(\mathbf{k}) /|w(\mathbf{k})|^{2}$ are strongest near the phase transition, Figs. 3a and $3 \mathrm{~d}$.

In contrast to what was assumed in the last paragraphs, optical lattices used in experiments are not homogeneous. Magnetic or optical trapping potentials are used to confine the atomic gas to a finite volume. Usually, this amounts to a parabolic trapping potential on top of the optical lattice. Consequently the lattice is in general not in a pure Mott or superfluid phase, but shows alternating shells of superfluid and Mott regions.

\section{Outlook and conclusions}

With this short review we intended to recall the connection between two apparently very different systems, Josephson junction arrays and bosons in optical lattices. Both systems are described by the Bose-Hubbard model. The prediction and experimental realization of a Mott transition in both systems has kindled interest in quantum phase transitions and triggered a great deal of theoretical and experimental work. 
On the theory side, in addition to what we discussed above we like to mention the proposal to study the coexistence of solid (DLRO) and superfluid (ODLRO) order in optical lattices [45]. Similar questions were studied in Josephson arrays in the past, see [46]. The superposition of a random laser speckle pattern and an optical lattice has been proposed as a system to study Anderson localization [47]. Mott states of bosonic atoms in an optical lattice have also been proposed as a starting point to create controlled multi-particle entanglement as an essential ingredient for quantum computation $[48,49,50]$. Fermionic atoms in optical lattices are also a very active research area. In Ref. [51], fermionic atoms in an optical lattice were described by a fermionic negative-U Hubbard model, and a BCS-transition and high-temperature superfluidity was predicted. Mixtures of fermions and Bosons were considered in [52], and a very complex phase diagram was found. The possibility to use a complex phase pattern of lattice lasers to create a "magnetic frustration" and observe fractional quantum Hall states was discussed in [53, 54, 55]. We finally mention the possibility to study optical lattices with spinor condensates [56] which present a very rich phase diagram.

On the experimental side, we like to mention the creation of highly entangled states by controlled shifts between two superposed optical lattices [57], and the observation of a TonksGirardeau gas of ultra-cold atoms in an optical lattice [58]. The transition from a strongly interacting 1D superfluid to a Mott insulator in a bosonic system has been observed [59], and for fermionic atoms in a 3D optical lattice Fermi surfaces were studied experimentally [60]. All of this shows that optical lattices form kind of a "many-body laboratory", and we expect a lot of interesting results and new phenomena in the years ahead both in the bosonic and in the fermionic case.

Acknowledgements It is a great pleasure and honor for us to be invited to write a contribution to celebrate Bernhard Mühlschlegel's 80th birthday. He has been one of the pioneers of the theory of superconductivity and, in particular, of the properties of Josephson junction arrays. His foresight led to a very stimulating and productive collaboration between one of his Ph.D. students, A. Kampf, and one of the authors (GS), which had a strong impact on the later work of both as well as on the research direction of the other two authors (CB and RF).

\section{References}

[1] For a number of articles see J.E. Mooij and G. Schön (eds.), Coherence in Superconducting Networks, Physica B 152 (1988).

[2] K.B. Efetov, Sov. Phys. JETP 51, 1015 (1980); R.M. Bradley and S. Doniach, Phys. Rev. B 30, 1138 (1984); S. Chakravarty, G.-L. Ingold, S. Kivelson, and A. Luther, Phys. Rev. Lett. 56, 2303 (1986); M.P.A. Fisher, Phys. Rev. B 36, 1917 (1987); W. Zwerger, J. Low Temp. Phys. 72, 291 (1988).

[3] A. Kampf and G. Schön, Phys. Rev. B 36, 3651 (1987); Phys. Rev. B 37, 5954 (1988).

[4] P. Fazekas, B. Mühlschlegel, and M. Schröter, Z. Phys. B 57, 193 (1984); B. Mühlschlegel and D. L. Mills, Phys. Rev. B 29, 159 (1984).

[5] M.P.A. Fisher, G. Grinstein, and S.M. Girvin, Phys. Rev. Lett. 64, 587 (1990).

[6] R. Fazio and G. Schön, Phys. Rev. B 43, 5307 (1991).

[7] R. Fazio and H. van der Zant, Phys. Rep. 355, 235 (2001).

[8] U. Eckern and A. Schmid, Phys. Rev. B 39, 6441 (1989).

[9] A. Minguzzi, S. Succi, F. Toschi, M.P. Tosi, and P. Vignolo, Phys. Rep. 395, 223 (2004).

[10] D. Jaksch, C. Bruder, J.I. Cirac, C.W. Gardiner, and P. Zoller, Phys. Rev. Lett. 81, 3108 (1998).

[11] M. Greiner, O. Mandel, T. Esslinger, T. W. Hänsch, and I. Bloch, Nature (London) 415, 39 (2002).

[12] M.P.A. Fisher, B.P. Weichman, G. Grinstein, and D.S. Fisher, Phys. Rev. B 40, 546 (1989).

[13] R.F. Voss and R.A. Webb, Phys. Rev. B 25, 3446 (1982); R.A. Webb, R.F. Voss, G. Grinstein, and P.M. Horn, Phys. Rev. Lett. 51, 690 (1983).

[14] M. Goldman and S.A. Wolf (eds.), Percolation, Localization, and Superconductivity, NATO ASI 108 (1986).

[15] H.A. Cerdeira and S.R. Shenoy (eds.), Josephson Junction Arrays, Physica B 222, pp 253-406 (1996). 
[16] L.J. Geerligs et al., Phys. Rev. Lett. 63, 326 (1989).

[17] R. Fazio and G. Schön, Phys. Rev. B 43, 5307 (1991).

[18] R. Fazio, A. van Otterlo, G. Schön, H.S.J. van der Zant, J.E. Mooij, Helv. Phys. Acta 65, 228 (1992).

[19] Ya.M. Blanter, and G. Schön, Phys. Rev. B 53, 14534 (1996).

[20] Ya.M. Blanter, R. Fazio, and G. Schön, Nucl. Phys. B S58, 79 (1997).

[21] J.E. Mooij, B.J. van Wees, L.J. Geerligs, M. Peters, R. Fazio, and G. Schön, Phys. Rev. Lett. 65, 645 (1990)

[22] R. Fazio, A. van Otterlo, and G. Schön, Europhys. Lett. 25, 453 (1994).

[23] U. Geigenmüller, C.J. Lobb and C.B. Whan, Phys. Rev. B 47, 348 (1993).

[24] H.S.J. van der Zant, F.C. Fritschy, T.P. Orlando, and J.E. Mooij, Phys. Rev. Lett. 66, 2531 (1991).

[25] T.S. Tighe, A.T. Jonson, and M. Tinkham, Phys. Rev. B 44, 10286 (1991).

[26] A. Matsuda and T. Kawakami, Phys. Rev. Lett. 51, 694 (1983).

[27] A. Fujimaki, K. Nakajima, and Y. Sawada, Phys. Rev. Lett. 59, 2985 (1987).

[28] H.S.J. van der Zant, T.P. Orlando, S. Watanabe, and S.H. Strogatz, Phys. Rev. Lett. 74, 174 (1995).

[29] S. Watanabe, S.H. Strogatz, H.S.J. van der Zant, and T.P. Orlando, Phys. Rev. Lett. 74, 379 (1995).

[30] H.S.J. van der Zant, F.C. Fritschy, T.P. Orlando, and J.E. Mooij, Europhys. Lett. 18, 343 (1992).

[31] E.B. Sonin, Phys. Rev. B 55, 485 (1997).

[32] M.P.A. Fisher, Physica A 177, 553 (1991).

[33] Yu.G. Makhlin and G.E. Volovik, Pis'ma Zh. Eksp. Teor. Fiz. 62, 923 (1985) [JETP Lett. 62, 941 (1995)].

[34] G. E. Volovik, cond-mat/9707136.

[35] There was some controversy in the literature on this point. It has been suggested that the offset charge in the equation of motion should be replaced with the electron number (F. Gaitan and S. R. Shenoy, Phys. Rev. Lett. 76, 4404 (1996)). We do not share this point of view since only the offset charges responsible for deviations from local charge neutrality in the array lead to the Magnus force.

[36] B.J. van Wees, H.S.J. van der Zant, and J.E. Mooij, Phys. Rev. B 35, 7291 (1987).

[37] P. Delsing, C.D. Chen, D.B. Haviland, T. Bergsten, and T. Claeson, in: Superconductivity in Networks and Mesoscopic Structures, edited by C. Giovannella and C.J. Lambert, Am. Institute of Physics (1997).

[38] W. Zwerger, J. Opt. B: Quantum Semiclass. Opt. 5, S9 (2003).

[39] L. Pitaevskii and S. Stringari, Phys. Rev. Lett. 87, 180402 (2001).

[40] A. Cuccoli, A. Fubini, R. Vaia, and V. Tognetti, Phys. Rev. A 64, 061601 (2001).

[41] R. Roth and K. Burnett, Phys. Rev. A 67, 031602(R) (2003).

[42] C. Schroll, F. Marquardt, and C. Bruder, Phys. Rev. A 70, 053609 (2004).

[43] J. K. Freericks and H. Monien, Phys. Rev. B 53, 2691 (1996); N. Elstner and H. Monien, condmat/9905367.

[44] The plots in Fig. 3 are obtained using the Bose-Hubbard Hamiltonian with an on-site interaction of the form $(\mathrm{U} / 2) \sum_{i} n_{i}\left(n_{i}-1\right)$ in order not to include the interaction if there is only one boson on a given site $[12]$.

[45] H. P. Büchler and G. Blatter, Phys. Rev. Lett. 91, 130404 (2003).

[46] C. Bruder, R. Fazio, and G. Schön, Phys. Rev. B 47, 342 (1993); A. van Otterlo, K.-H. Wagenblast, R. Baltin, C. Bruder, R. Fazio, and G. Schön, Phys. Rev. B 52, 16176 (1995).

[47] B. Damski, J. Zakrzewski, L. Santos, P. Zoller, M. Lewenstein, Phys. Rev. Lett. 91, 080403 (2003).

[48] D. Jaksch, H.-J. Briegel, J. I. Cirac, C. W. Gardiner, and P. Zoller, Phys. Rev. Lett. 82, 1975 (1999).

[49] O. Mandel, M. Greiner, A. Widera, T. Rom, T. W. Hänsch, and I. Bloch, Nature (London) 425, 937 (2003).

[50] H.-J. Briegel and R. Raussendorf, Phys. Rev. Lett. 86, 910 (2001).

[51] W. Hofstetter, J. I. Cirac, P. Zoller, E. Demler, and M. D. Lukin, Phys. Rev. Lett. 89, 220407 (2002).

[52] M. Lewenstein, L. Santos, M. A. Baranov, and H. Fehrmann, Phys. Rev. Lett. 92, 050401 (2004).

[53] A.S. Sorensen, E. Demler, and M.D. Lukin, Phys. Rev. Lett. 94, 086803 (2005).

[54] D. Jaksch and P. Zoller, New J. Phys. 5, 56 (2003).

[55] M. Polini, R. Fazio, A. H. MacDonald, and M. P. Tosi, Phys. Rev. Lett. (in press); cond-mat/0501387.

[56] E. Demler and F. Zhou, Phys. Rev. Lett. 88, 163001 (2002).

[57] O. Mandel, M. Greiner, A. Widera, T. Rom, T.W. Hänsch, and I. Bloch, Nature 425, 937 (2003).

[58] B. Paredes, A. Widera, V. Murg, O. Mandel, S. Fölling, I. Cirac, G.V. Shlyapnikov, T. Rom, T.W. Hänsch, and I. Bloch, Nature 429, 277 (2004).

[59] T. Stöferle, H. Moritz, C. Schori, M. Köhl, and T. Esslinger, Phys. Rev. Lett. 92, 130403 (2004).

[60] M. Köhl, H. Moritz, T. Stöferle, K. Günter, and T. Esslinger, Phys. Rev. Lett. 94, 080403 (2005). 